International Journal of Pure and Applied Mathematics

Volume 92 No. 1 2014, 61-71

ISSN: 1311-8080 (printed version); ISSN: 1314-3395 (on-line version)

url: http://www.ijpam.eu

doi: http://dx.doi.org/10.12732/ijpam.v92i1.5

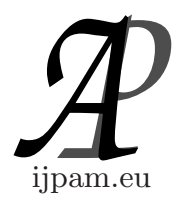

\title{
EXISTENCE OF WEAK SOLUTIONS \\ FOR $p(x)$-KIRCHHOFF-TYPE EQUATION
}

\author{
Zehra Yucedag \\ Department of Mathematics \\ Faculty of Science \\ Dicle University \\ 21280, Diyarbakir, TURKEY
}

\begin{abstract}
This paper is considerned with the existence of solutions for $p(x)$ Kirchhoff-type problem under with Dirichlet boundary condition. By direct variational method and the Mountain Pass theorem, we establish some conditions that ensure the existence nontrivial weak solutions for the problem.
\end{abstract}

AMS Subject Classification: 35D05, 35J60, 35J70

Key Words: variational method, $p(x)$-Kirchhoff-type equation, Mountain Pass Theorem

\section{Introduction}

In this paper we are concerned with the following problem

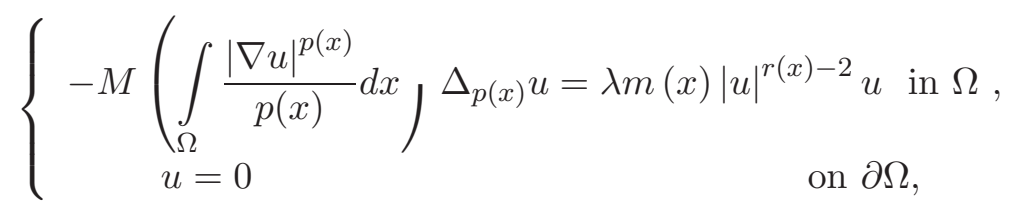

where $\Omega \subset \mathbb{R}^{N}(N \geq 2)$ is a smooth bounded domain, $p, r, s \in C(\bar{\Omega})$ for any $x \in \bar{\Omega} ; m$ is a non-negative measurable real function.

We assume that $M$ and $m$ are satisfy the following conditions specief conditions.

Received: November 13, 2013

(C) 2014 Academic Publications, Ltd. url: www.acadpubl.eu 
$\left(M_{1}\right) M: \mathbb{R}^{+} \rightarrow \mathbb{R}^{+}$is a continuous function such that

$$
m_{1} t^{\alpha(x)-1} \leq M(t) \leq m_{2} t^{\alpha(x)-1}
$$

for all $t>0$ and $m_{1}, m_{2}, \alpha$ real numbers such that $0<m_{1} \leq m_{2}$ and $\alpha(x) \geq 1$;

(A1) $m \in L^{\beta(x)}(\Omega), M(x)>0$ and $\beta \in C_{+}(\bar{\Omega})$ such that $\frac{1}{\beta(x)}+\frac{1}{\beta_{0}(x)}=1$, $p(x)<\frac{\beta(x)-1}{\beta(x)} p^{*}(x)$ and $1<r(x)<\frac{\beta(x)}{\beta(x)-1}, \forall x \in \bar{\Omega}$;

(A2) $1<r^{-} \leq r^{+}<\alpha^{-} p^{-}<\alpha^{+} p^{+}$and $\alpha^{+} p^{+}<\left(p^{*}\right)^{-}$, where $\left(p^{*}\right)^{-}=$ $\frac{N p^{-}}{N-p^{-}}$.

Problem $(\mathbf{P})$ is related to the stationary version of a model, the so-called Kirchhoff equation, introduced by Kirchhoff [16]. To be more precise, Kirchhoff established a model given by the equation

$$
\rho \frac{\partial^{2} u}{\partial t^{2}}-\left(\frac{P_{0}}{h}+\frac{E}{2 L} \int_{0}^{L}\left|\frac{\partial u}{\partial x}\right|^{2} d x\right) \frac{\partial^{2} u}{\partial x^{2}}=0,
$$

where $\rho, P_{0}, h, E, L$ are constants, which extends the classical D'Alambert's wave equation, by considering the effects of the changes in the length of the strings during the vibrations.

In recent years, elliptic problems involving $p$-Kirchhoff and $p(x)$-Kirchhoff type operators have been studied in many papers, we refer to $[2,5,7,8,9,10$, $18,23,25]$.

The $p(x)$-Laplacian operator $\Delta_{p(x)} u=\operatorname{div}\left(|\nabla u|^{p(x)-2} \nabla u\right)$ is a generalization of $p$-Laplace operator $\Delta_{p} u=\operatorname{div}\left(|\nabla u|^{p-2} \nabla u\right)$ where $p>1$ is a real constant. The $p(x)$-Laplacian possesses more complicated structure than the $p$-Laplacian; for example, it is not homogeneous.This fact implies some diffculties; for example, we can not use the theory of Sobolev spaces in many problems involving this operator. Some of the nonlinear problems involving $p(x)$-growth conditions are extremely attractive because those problems can be used to model dynamical phenomenons that arise from the study of electrorheological fluids or elastic mechanics [1, 11, 15, 22, 26]. Moreover, problems with variable exponent growth conditions also appear in the mathematical modelling of stationary thermo-rheological viscous flows of non-Newtonian fluids, in the mathematical description of the processes filtration of an ideal barotropic gas through a porous medium and image processing $[3,4,6]$. The detailed application backgrounds of the $p(x)$-Laplace operator can be found in $[13,14,20,21,17]$. 
In the present paper, by help of the Mountain Pass theorem, we obtain the existence at least one nontrivial weak solution of problem $(\mathbf{P})$. This paper is organized as follows. In Section 2, we present some necessary preliminary knowledge on variable exponent Sobolev spaces. In Section 3, we show the existence of weak solutions of problem $(\mathbf{P})$.

\section{Preliminaries}

We state some definitions and basic properties of variable exponent LebesgueSobolev spaces $L^{p(x)}(\Omega), W^{1, p(x)}(\Omega), W_{0}^{1, p(x)}(\Omega)$ and $L_{c(x)}^{p(x)}(\Omega)$ ( for details, see $[13,17,19])$.

Set

$$
C_{+}(\bar{\Omega})=\{p ; p \in C(\bar{\Omega}), p(x)>1, \text { for any } x \in \bar{\Omega}\} .
$$

For any $p(x) \in C_{+}(\bar{\Omega})$, we denote

$$
1<p^{-}:=\min _{x \in \bar{\Omega}} p(x) \leq p(x) \leq p^{+}:=\max _{x \in \bar{\Omega}} p(x)<\infty .
$$

For any $p(x) \in C_{+}(\bar{\Omega})$, we define the variable exponent Lebesgue space by

$$
L^{p(x)}(\Omega)=\left\{u \mid u: \Omega \rightarrow \mathbb{R} \text { is measurable, } \int_{\Omega}|u(x)|^{p(x)} d x<\infty\right\} .
$$

The modular of the $L^{p(x)}(\Omega)$ space, which is the mapping $\rho_{p(x)}: L^{p(x)}(\Omega) \rightarrow \mathbb{R}$ defined by

$$
\rho_{p(x)}(u)=\int_{\Omega}|u(x)|^{p(x)} d x, \forall u \in L^{p(x)}(\Omega) .
$$

Proposition 2.1. (see $[13,17]$ ) The conjugate space of $L^{p(x)}(\Omega)$ is $L^{p^{\prime}(x)}(\Omega)$, where $\frac{1}{p^{\prime}(x)}+\frac{1}{p(x)}=1$. For any $u \in L^{p(x)}(\Omega)$ and $v \in L^{p^{\prime}(x)}(\Omega)$, we have

$$
\left|\int_{\Omega} u v d x\right| \leq\left(\frac{1}{p^{-}}+\frac{1}{\left(p^{-}\right)^{\prime}}\right)|u|_{p(x)}|v|_{p^{\prime}(x)} .
$$

Proposition 2.2. (see $[13,17]$ ) If $u, u_{n} \in L^{p(x)}(\Omega), n=1,2, \ldots$ we have

$$
(i)|u|_{p(x)}>1 \Longrightarrow|u|_{p(x)}^{p^{-}} \leq \rho_{p(x)}(u) \leq|u|_{p(x)}^{p^{+}} ;
$$




$$
\begin{aligned}
& (i i)|u|_{p(x)}<1 \Longrightarrow|u|_{p(x)}^{p^{+}} \leq \rho_{p(x)}(u) \leq|u|_{p(x)}^{p^{-}} \\
& (\text {iii }) \lim _{n \rightarrow \infty}\left|u_{n}\right|_{p(x), \Omega}=0 \Leftrightarrow \lim _{n \rightarrow \infty} \rho_{p(x)}\left(u_{n}\right)=0
\end{aligned}
$$

The variable exponent Sobolev space $W^{1, p(x)}(\Omega)$ is denined by

$$
W^{1, p(x)}(\Omega)=\left\{u \in L^{p(x)}(\Omega)|| \nabla u \mid \in L^{p(x)}(\Omega)\right\},
$$

with the norm

$$
\|u\|_{1, p(x)}=|u|_{p(x)}+|\nabla u|_{p(x)}, \forall u \in W^{1, p(x)}(\Omega) .
$$

The space $W_{0}^{1, p(x)}(\Omega)$ is denoted by the closure of $C_{0}^{\infty}(\Omega)$ in $W^{1, p(x)}(\Omega)$ with respect to the norm $\|u\|_{1, p(x)}$. We can define an equivalent norm

$$
\|u\|_{p(x)}=|\nabla u|_{p(x)} .
$$

Proposition 2.3. (see [12]) Let $p(x)$ and $q(x)$ be measurable functions such that $p(x) \in L^{\infty}(\Omega)$, and $1 \leq p(x) q(x) \leq \infty$, for a.e. $x \in \Omega$. Let $u \in L^{q(x)}(\Omega)$, $u \neq 0$. Then

$$
\begin{gathered}
|u|_{p(x) q(x)} \leq 1 \Longrightarrow|u|_{p(x) q(x)}^{p^{+}} \leq\left.\left.|| u\right|^{p(x)}\right|_{q(x)} \leq|u|_{p(x) q(x)}^{p^{-}} \\
|u|_{p(x) q(x)} \geq 1 \Longrightarrow|u|_{p(x) q(x)}^{p^{-}} \leq\left.\left.|| u\right|^{p(x)}\right|_{q(x)} \leq|u|_{p(x) q(x)}^{p^{+}}
\end{gathered}
$$

In particular, if $p(x)=p$ is constant, then $\left.\left.|| u\right|^{p}\right|_{q(x)}=|u|_{p q(x)}^{p}$.

We also consider the weighted variable exponent Lebesgue space $L_{c(x)}^{p(x)}(\Omega)$. Let $c: \Omega \rightarrow \mathbb{R}$ be a measurable real function such that $c(x)>0$ a.e. $x \in \Omega$. We define

$$
L_{c(x)}^{p(x)}(\Omega)=\left\{u \mid u: \Omega \rightarrow \mathbb{R} \text { is measurable, } \int_{\Omega} c(x)|u(x)|^{p(x)} d x<\infty ; c(x)>0\right\},
$$

The then $L_{c(x)}^{p(x)}(\Omega)$ is a Banach space which has similar properties with the usual variable exponent Lebesgue spaces. The modular of this space is $\rho_{(c(x), p(x))}$ : $L_{c(x)}^{p(x)}(\Omega) \rightarrow \mathbb{R}$ defined by

$$
\rho_{(c(x), p(x))}(u)=\int_{\Omega} c(x)|u(x)|^{p(x)} d x
$$


Proposition 2.4. (see [17]) If $p^{+}<\infty$ and $u, u_{n} \in L_{c(x)}^{p(x)}(\Omega), n=1,2, \ldots$ we have

$(i)|u|_{(c(x), p(x))}>1 \Longrightarrow|u|_{(c(x), p(x))}^{p^{-}} \leq \rho_{(c(x), p(x))}(u) \leq|u|_{(c(x), p(x))}^{p^{+}}$,

$(i i)|u|_{(c(x), p(x))}<1 \Longrightarrow|u|_{(c(x), p(x))}^{p^{+}} \leq \rho_{(c(x), p(x))}(u) \leq|u|_{(c(x), p(x))}^{p^{-}}$,

(iii) $\lim _{n \rightarrow \infty}\left|u_{n}\right|_{(c(x), p(x))}=0 \Leftrightarrow \lim _{n \rightarrow \infty} \rho_{(c(x), p(x))}\left(u_{n}\right)=0$.

Proposition 2.5. (see $[13,17]$ ) (i) If $1<p^{-} \leq p^{+}<\infty$ then, the spaces $L^{p(x)}(\Omega), W^{1, p(x)}(\Omega)$ and $W_{0}^{1, p(x)}(\Omega)$ are separable and reflexive Banach spaces;

(ii) Let $q \in C_{+}(\bar{\Omega})$.If $q(x)<p^{*}(x)$, for all $x \in \bar{\Omega}$, then the embedding $W_{0}^{1, p(x)}(\Omega) \hookrightarrow L^{q(x)}(\Omega)$ is compact and continuous $\left(p^{*}(x)=\frac{N p(x)}{N-p(x)}\right.$ if $N>$ $p(x)$ or $p^{*}(x)=\infty$ if $\left.N \leq p(x)\right)$, also there is a constant $c>0$ such that

$$
|u|_{q(x)} \leq c\|u\|, \text { for all } u \in W_{0}^{1, p(x)}(\Omega) .
$$

Proposition 2.6. (see [14]) Let $X$ be a Banach space and

$$
\Lambda(u)=\int_{\Omega} \frac{|\nabla u|^{p(x)}}{p(x)} d x .
$$

The functional $\Lambda: X \rightarrow \mathbb{R}$ is convex. The mapping $\Lambda^{\prime}: X \rightarrow X^{*}$ is a strictly monotone, bounded homeomorphism, and of $\left(S_{+}\right)$type, namely

$$
u_{n} \rightarrow u\left(\text { weakly) and } \varlimsup_{n \rightarrow \infty}\left\langle\Lambda^{\prime}\left(u_{n}\right), u_{n}-u\right\rangle \leq 0 \text { implies } u_{n} \rightarrow u\right. \text { (strongly), }
$$

where $X=W_{0}^{1, p(x)}(\Omega)$.

\section{Main Results}

We say that $u \in X$ is a weak solution of $(\mathbf{P})$ if

$$
M\left(\int_{\Omega} \frac{|\nabla u|^{p(x)}}{p(x)} d x\right) \int_{\Omega}|\nabla u|^{p(x)-2} \nabla u \nabla \varphi d x=\lambda \int_{\Omega} m(x)|u|^{r(x)-2} u \varphi d x,
$$

where $\varphi \in X$. 
We associate to the problem $(\mathbf{P})$ the energy functional, defined as $I: X \rightarrow$ $\mathbb{R}$,

$$
I(u)=\widehat{M}\left(\int_{\Omega} \frac{|\nabla u|^{p(x)}}{p(x)} d x J-\lambda \int_{\Omega} \frac{m(x)}{r(x)}|u|^{r(x)} d x,\right.
$$

where $\widehat{M}(t)=\int_{0}^{t} M(s) d s$. In a standart way, it can be shown that $I \in$ $C^{1}(X, \mathbb{R})$.Moreover, we have

$$
\begin{aligned}
\left\langle I^{\prime}(u), v\right\rangle=M\left(\int_{\Omega} \frac{|\nabla u|^{p(x)}}{p(x)} d x\right) \int_{\Omega}|\nabla u|^{p(x)-2} \nabla u \nabla v d x & -\lambda \int_{\Omega} m(x)|u|^{r(x)-2} u v d x,
\end{aligned}
$$

for any $u, v \in X$. Hence, we can infer that critical points of functional $I$ are the weak solutions for problem $(\mathbf{P})$.

Theorem 3.1. Assume that the conditions $\left(M_{1}\right),(\mathbf{A 1})$ and (A2) are satisfied, then there exists $\lambda^{*}>0$ such that for any $\lambda \in\left(0, \lambda^{*}\right), \operatorname{problem}(\mathbf{P})$ has a nontrivial weak solution.

Definition 3.2. We say that $I$ satisfies Palais-Smale condition in $X((P S)$ condition for short) if every sequence $\left\{u_{n}\right\} \subset X$ such that $\left|I\left(u_{n}\right)\right| \leq c$ and $I^{\prime}\left(u_{n}\right) \rightarrow 0$ contains a convergent subsequence in the norm of $X$.

Lemma 3.3. Assume that $\left(M_{1}\right),(\mathbf{A 1})$ and (A2) hold. There exist two positive real numbers $\rho$ and $\gamma$ such that for any $\lambda \in\left(0, \lambda^{*}\right)$, we have

$$
I(u) \geq \rho>0, \forall u \in X \text { with }\|u\|=\gamma .
$$

Proof. By using the conditions (A2) and (A1), the embedding from $X$ to $L_{m(x)}^{r(x)}(\Omega)$ is compact (see Theorem $2.8[19]$ ). Then, we can write

$$
\int_{\Omega} m(x)\left|u_{n}\right|^{r(x)} d x \leq C\left(\left\|u_{n}\right\|^{r^{+}}+\left\|u_{n}\right\|^{r^{-}}\right),
$$

for all $u \in X$. We consider $\|u\|<1$. Then, by $\left(M_{1}\right)$, (3.1) and Proposition 2.2, we have

$$
I(u) \geq \frac{m_{1}}{\alpha^{+}\left(p^{+}\right)^{\alpha^{+}}}\|u\|^{\alpha^{+} p^{+}}-\frac{\lambda}{r^{-}} \int_{\Omega} m(x)|u|^{r(x)} d x
$$




$$
\begin{aligned}
& \geq \frac{m_{1}}{\alpha^{+}\left(p^{+}\right)^{\alpha^{+}}}\|u\|^{\alpha^{+} p^{+}}-\frac{\lambda c_{2}}{r^{-}}\|u\|^{r^{-}} \\
& =\left(\frac{m_{1}}{\alpha^{+}\left(p^{+}\right)^{\alpha^{+}}}\|u\|^{\alpha^{+} p^{+}-r^{-}}-\frac{\lambda c_{2}}{r^{-}}\right)\|u\|^{r^{-}} .
\end{aligned}
$$

By the above inequality, if we choose

$$
\lambda^{*}=\frac{m_{1} r^{-}}{2 \alpha^{+}\left(p^{+}\right)^{\alpha^{+}} c_{2}} \gamma^{\alpha^{+} p^{+-r^{-}}},
$$

then, there exist two positive real numbers $\rho$ and $\gamma$ such that $\lambda \in\left(0, \lambda^{*}\right)$, we have

$$
I(u) \geq \rho>0, \forall u \in W_{0}^{1, p(x)}(\Omega) \text { with }\|u\|=\gamma \in(0,1) .
$$

The proof of Lemma 3.3 is complete.

Lemma 3.4. Assume that $\left(M_{1}\right),(\mathbf{A 1})$ and (A2) hold. Then, there exists $\omega \in X$ such that $\omega \geq 0, \omega \neq 0$ and $I(t \omega)<0$ for all $t>0$ small enough.

Proof. Then by $\left(M_{1}\right)$, we have

$$
\begin{aligned}
I(t \omega) & =\widehat{M}\left(\int_{\Omega} \frac{|\nabla t \omega|^{p(x)}}{p(x)} d x\right)^{-\lambda} \int_{\Omega} \frac{m(x)}{r(x)}|t \omega|^{r(x)} d x \\
& \leq \frac{m_{2} t^{\alpha^{-} p^{-}}}{\alpha^{-}\left(p^{-}\right)^{\alpha^{-}}}\left(\int_{\Omega}|\nabla \omega|^{p(x)} d x\right)-\frac{\lambda t^{r+}}{r^{+}} \int_{\Omega} m(x)|\omega|^{r(x)} d x
\end{aligned}
$$

Thus,

$$
I(t \omega)<0
$$

for all $t<\sigma^{\frac{1}{\alpha^{-} p^{-}-r^{+}}}$with

$$
0<\sigma<\min \left\{1, \frac{\lambda \alpha^{-}\left(p^{-}\right)^{\alpha^{-}} \int_{\Omega} m(x)|\omega|^{r(x)} d x}{m_{2} r^{+}\left(\int_{\Omega}|\nabla \omega|^{p(x)} d x\right)^{\alpha^{-}}}\right\} .
$$

The proof of Lemma 3.4 is complete. 
Lemma 3.5. Assume that $\left(M_{1}\right),(\mathbf{A 1})$ and (A2) hold. Then, I satisfies $(P S)$ condition.

Proof. Let assume that there exists a sequence $\left\{u_{n}\right\} \subset X$ such that

$$
\left|I\left(u_{n}\right)\right| \leq c \text { and } I^{\prime}\left(u_{n}\right) \rightarrow 0 \text { as } n \rightarrow \infty .
$$

Firstly, we prove that $\left\{u_{n}\right\}$ is bounded in $X$. Arguing by contradiction and passing to a subsequence, we have $\left\|u_{n}\right\| \rightarrow \infty$ as $n \rightarrow \infty$. Using $\left(M_{1}\right),(3.2)$ and considering $\left\|u_{n}\right\|>1$, for $n$ large enough, we have

$$
\begin{aligned}
c & \geq I\left(u_{n}\right) \geq \frac{m_{1}}{\alpha^{+}\left(p^{+}\right)^{\alpha^{+}}}\left(\int_{\Omega}\left|\nabla u_{n}\right|^{p(x)} d x\right)-\frac{\lambda}{r^{-}} \int_{\Omega} m(x)\left|u_{n}\right|^{r(x)} d x \\
& \geq \frac{m_{1}}{\alpha^{+}\left(p^{+}\right)^{\alpha^{+}}}\left\|u_{n}\right\|^{\alpha^{-} p^{-}}-\frac{\lambda c_{1}}{r^{-}}\left\|u_{n}\right\|^{r^{+}} .
\end{aligned}
$$

Since $r^{+}<\alpha^{-} p^{-}$, we obtain that $\left\{u_{n}\right\}$ is bounded in $X$. Therefore, there exists a subsequence, again denoted by $\left\{u_{n}\right\}$ and $u \in X$ such that $u_{n} \rightarrow u$ in $X$. By $(3.2)$, we have $\left\langle I^{\prime}\left(u_{n}\right), u_{n}-u\right\rangle \rightarrow 0$.

$$
\begin{aligned}
& \left\langle I^{\prime}\left(u_{n}\right), u_{n}-u\right\rangle= \\
& M\left(\int_{\Omega} \frac{\left|\nabla u_{n}\right|^{p(x)}}{p(x)} d x\right) \int_{\Omega}\left|\nabla u_{n}\right|^{p(x)-2} \nabla u_{n}\left(\nabla u_{n}-\nabla u\right) \\
& -\lambda \int_{\Omega} m(x)\left|u_{n}\right|^{r(x)-2} u_{n}\left(u_{n}-u\right) d x \rightarrow 0 .
\end{aligned}
$$

On the other hand, using Proposition 2.1-2.3-2.7 and the compact embedding $\left(X \hookrightarrow L_{m(x)}^{r(x)}(\Omega)\right)$, we have

$$
\int_{\Omega} m(x)\left|u_{n}\right|^{r(x)-2} u_{n}\left(u_{n}-u\right) d x \rightarrow 0 .
$$

Therefore, we obtain

$$
M\left(\int_{\Omega} \frac{\left|\nabla u_{n}\right|^{p(x)}}{p(x)} d x\right) \int_{\Omega}\left|\nabla u_{n}\right|^{p(x)-2} \nabla u_{n}\left(\nabla u_{n}-\nabla u\right) \rightarrow 0 .
$$


In view of $\left(M_{1}\right)$, we conclude that

$$
\int_{\Omega}\left|\nabla u_{n}\right|^{p(x)-2} \nabla u_{n}\left(\nabla u_{n}-\nabla u\right) \rightarrow 0 .
$$

Thus, from Proposition 2.6, it follows that $u_{n} \rightarrow u$ in $W_{0}^{1, p(x)}(\Omega)$. I satisfies $(P S)$ condition.

Proof of Theorem 3.1, from Lemma 3.3, Lemma 3.4, Lemma 3.5 and the fact that $I(0)=0, I$ satisfies the Mountain Pass theorem [24]. Therefore, $I$ has at least one nontrivial weak solution.

\section{References}

[1] Acerbi E, Mingione G. Regularity results for stationary electro-rheological fluids. Arch Ration Mech Anal 2002; 164: 213-259.

[2] C. O. Alves, F. J. S. A. Corrêa and T. F. Ma, Positive Solutions for a Quasilinear Elliptic Equation of Kirchhoff Type, Computers and Mathematics with Appl. 49 (2005), 85-93.

[3] Antontsev SN, Rodrigues JF. On stationary thermo-rheological viscous flows. Ann Univ Ferrara Sez VII Sci Mat 2006; 52: 19-36.

[4] Antontsev SN, Shmarev SI. A model porous medium equation with variable exponent of nonlinearity: existence, uniqueness and localization properties of solutions. Nonlinear Anal 2005; 60: 515-545.

[5] M. Avci, B. Cekic and R. A. Mashiyev, Existence and multiplicity of the solutions of the $p(x)$-Kirchhoff type equation via genus theory, Math. Methods in the Applied Sciences, 34 (2011), no.14,1751-1759.

[6] C.Y. Chen, Kuo and T.F.Wu, The Nehari manifold for a Kirchhoff type problem involving sign-changing weight functions, J. Differential Equations, 250 (2011),1876-1908.

[7] B. Cheng, X. Wu and J. Liu, Multiplicity of nontrivial solutions for Kirchhoff type problems, Boundary Value Problems, Vol. 2010, Article ID 268946, 13 pages.

[8] F. J. S. A. Corrêa and G.M. Figueiredo, On a elliptic equation of $p$-kirchhoff type via variational methods, Bull. Austral. Math. Soc. 74 (2006), 263-277. 
[9] F. J. S. A. Corrêa and G.M. Figueiredo, On a $p$-Kirchhoff equation via Krasnoselskii's genus, Appl. Math. Letters, 22 (2009), 819-822.

[10] G. Dai and R. Hao, Existence of solutions for a $p(x)$-Kirchhoff-type equation, J. Math. Anal. Appl. 359 (2009), 275-284.

[11] Diening L. Theoretical and Numerical Results for Electrorheological Fluids. Ph D thesis, University of Frieburg, Germany, 2002.

[12] Edmunds D, Rakosnik J. Sobolev embeddings with variable exponent. Studia Math, 2000; 143 (3): 267-293.

[13] X. L. Fan, J. S. Shen and D. Zhao, Sobolev embedding theorems for spaces $W^{k, p(x)}(\Omega)$, J. Math. Anal. Appl. 262 (2001), 749-760.

[14] X. L. Fan and Q. H. Zhang, Existence of solutions for $p(x)$-Laplacian Dirichlet problems, Nonlinear Anal. 52 (2003),1843-1852.

[15] T. C. Halsey, Electrorheological fluids, Science, 258 (1992), 761-766.

[16] G. Kirchhoff, Mechanik, Teubner, Leipzig, 1883.

[17] O. Kovăčik and J. Răkosnik, On spaces $L^{p(x)}$ and $W^{k, p(x)}$, Czechoslovak Math. J. 41 (1991), no.116, 592-618.

[18] D. Liu, On a $p$-Kirchhoff equation via fountain theorem and dual fountain theorem, Nonlinear Anal. 72 (2010), 302-308.

[19] R. A. Mashiyev, S. Ogras, Z. Yucedag and M. Avci, Existence and multiplicity of weak solutions for nonuniformly elliptic equations with nonstandard growth condition, Complex Variables and Elliptic Equations, 57 (2012), No. 5, 579-595.

[20] Mihăilescu M, Rădulescu V. On a nonhomogeneous quasilinear eigenvalue problem in Sobolev spaces with variable exponent. Proc Amer Math Soc, 2007; 135 (9): 2929-2937.

[21] Mihăilescu M, Rădulescu V. A multiplicity result for a nonlinear degenerate problem arising in the theory of electrorheological fluids. Proc R Soc Lond Ser A Math Phys Eng Sci 2006; 462 (2073): 2625-2641.

[22] M. Růžička, Electrorheological fluids: Modeling and Mathematical Theory, Lecture Notes in Mathematics, 1748, Springer-Verlag, Berlin, 2000. 
[23] J.J. Sun and C.L. Tang, Existence and multiplicity of solutions for Kirchhoff type equations, Nonlinear Anal. 74 (2011),1212-1222.

[24] M. Willem, Minimax Theorems, Birkhauser, Basel, 1996.

[25] Z. Yucedag, M. Avci and R. A. Mashiyev,On an Elliptic System of $p(x)$-Kirchhoff-type under Neumann Boundary Condition, Math. Model. and Anal. 17 (2012), no. 2,161-170.

[26] V. V. Zhikov, Averaging of functionals of the calculus of variations and elasticity theory, Math. USSR. Izv. 9 (1987), 33-66. 
